\title{
THE NUMBER OF LIMIT CYCLES FOR A CLASS OF QUINTIC HAMILTONIAN SYSTEMS UNDER QUINTIC PERTURBATIONS
}

\author{
GUOWEI CHEN, YONGBIN WU and XINAN YANG
}

(Received 23 June 1999; revised 28 August 2001)

Communicated by C. F. Miller

\begin{abstract}
The Hopf bifurcation and homoclinic bifurcation of the quintic Hamiltonian system is analyzed under quintic perturbations by using unfolding theory in this paper. We show that a quintic system can have at least 29 limit cycles.
\end{abstract}

2000 Mathematics subject classification: primary $34 \mathrm{C} 07,37 \mathrm{C} 10$.

Keywords and phrases: quintic Hamiltonian system, limit cycle, bifurcation.

\section{Introduction}

At the beginning of the 20th century Hilbert [4] posed 23 famous problems; the 16th problem remains unsolved. This problem consists of two parts. The absorbing interest is in the second part, which is concerned with the number and relative location of the limit cycles to a planar polynomial differential system

$$
\mathrm{d} x / \mathrm{d} t=P_{n}(x, y), \quad \mathrm{d} y / \mathrm{d} t=Q_{n}(x, y),
$$

where $P_{n}(x, y)$ and $Q_{n}(x, y)$ are polynomials in $x, y$ with degree $n$. The first major contribution is due to Dulac [3]. He proved that the number of limit cycles to system (1.1) is finite although his proof was recently discovered to be incomplete. For $n=2$, namely, for quadratic system, Shi [6], Chen and Wang [1] independently gave an example which shows that (1.1) has at least four limit cycles with the so called (3-1) distribution. Li [5], using a perturbation of a Hamiltonian system, constructed a cubic system which has at least 11 limit cycles. Recently, Zoladek [9] showed 


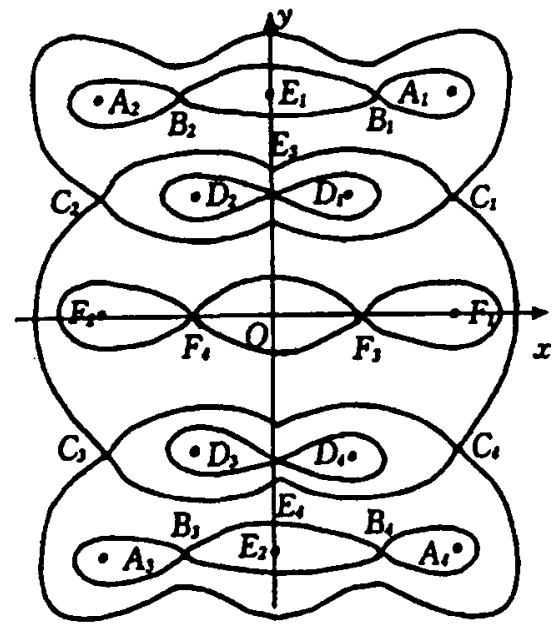

FiguRE 1.

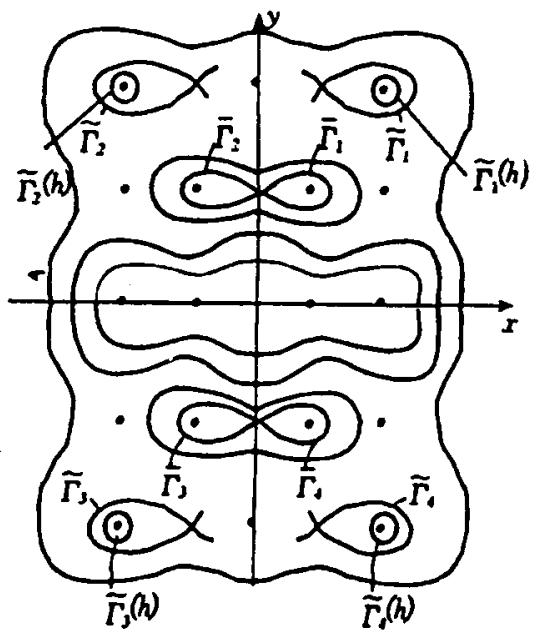

FIGURE 2.

that a cubic system can generate 11 limit cycles with small amplitude from the neighbourhood of a centre by slightly perturbing its parameters. In this paper we construct a quintic Hamiltonian system which can generate 29 limit cycles under some perturbation of a quintic polynomial. The distribution diagram of these limit cycles is also given. Our result ensures that a quintic polynomial differential system has at least 29 limit cycles.

The idea of constructing quintic perturbed system which can generate 29 limit cycles begins with the quintic Hamiltonian system

$$
\mathrm{d} x / \mathrm{d} t=y\left(1-y^{2} / 2\right)\left(1-y^{2} / 8\right), \quad \mathrm{d} y / \mathrm{d} t=-x\left(1-2 x^{2}\right)\left(1-x^{2} / 2\right) .
$$

System (1.2) has 25 critical points and has Hamiltonian functions

$$
H(x, y)=x^{2} / 2+y^{2} / 2-5 x^{4} / 8-5 y^{4} / 32+x^{6} / 6+y^{6} / 96 .
$$

The graph of $H(x, y)=h$, according to the value of $h$, can be sketched as in Figure 1, where $A_{i}, B_{i}, C_{i}, D_{i}, E_{i}, F_{i}, i=1,2,3,4$, and $O$ are critical points of system (1.2).

Consider a perturbed system

$$
\begin{aligned}
\mathrm{d} x / \mathrm{d} t & =y\left(1-y^{2} / 2\right)\left(1-y^{2} / 8\right)=P_{\varepsilon}(x, y), \\
\mathrm{d} y / \mathrm{d} t & =-x\left(1-2 x^{2}\right)\left(1-x^{2} / 2\right)+\varepsilon\left(\delta y+\mu x^{2} y+r y^{3}+k x^{4} y+n x^{2} y^{3}+y^{5}\right) \\
& =Q_{\varepsilon}(x, y)
\end{aligned}
$$

as a small disturbance of Hamiltonian system (1.2), where $0<|\varepsilon| \ll 1$. Without loss of generality we will assume that $0<\varepsilon \ll 1$. System (1.4) also has 25 critical points 
$\tilde{A}_{i}, \tilde{B}_{i}, \tilde{C}_{i}, \tilde{D}_{i}, \tilde{E}_{i}, \tilde{F}_{i}, i=1,2,3,4$, and $O$, each of which lies near $A_{i}, B_{i}, C_{i}, D_{i}, E_{i}$ and $F_{i}$, respectively ( $O$ is unchanged). We claim that system (1.4) has the following properties:

(i) $\tilde{A}_{1}, \tilde{A}_{2}, \tilde{A}_{3}$ and $\tilde{A}_{4}$ are fine foci with first order;

(ii) $\tilde{D}_{1}, \tilde{D}_{2}, \tilde{D}_{3}$ and $\tilde{D}_{4}$ are fine foci with second order.

It is well known that the number of limit cycles to a perturbed system can be determined by the zeros of Mel'nikov function (Chow [2]). The Mel'nikov function corresponding to system (1.4) is

$$
M(h)=\oint_{\Gamma_{h}}\left(\delta y+\mu x^{2} y+r y^{3}+k x^{4} y+n x^{2} y^{3}+y^{5}\right) \mathrm{d} x,
$$

where $\Gamma_{h}$ is a compact component of $H(x, y)=h$. If $M\left(h^{\prime}\right)=0$, then there are limit cycles of system (1.4) in the neighbourhood of $\Gamma_{h^{\prime}}$. If $\Gamma_{h}$ is a homoclinic loop of system (1.2), then the validity of $M(h)=0$ also shows that there exists a separatrix loop of system (1.4) which passes through a saddle point near $\Gamma_{h}$.

Using this reasoning we first find the zeros of $M(h)$, consequently, we obtain 5 limit cycles and 8 homoclinic loops of system (1.4), their distributions shown in Figure 2, where $\tilde{\Gamma}_{i}^{(h)}$ is a limit cycle surrounding $\tilde{A}_{i}, i=1,2,3,4 ; \tilde{\Gamma}_{i}$ is the homoclinic loop which passes $\tilde{B}_{i}$ and surrounds $\tilde{A}_{i}, i=1,2,3,4$; while $\bar{\Gamma}_{i}$ is the other homoclinic loop which passes $E_{j}$ and surrounds $D_{i}$, where $i=1,2$ for $j=1$ and $i=3,4$ for $j=2$.

One will find that the inner stability of $\tilde{\Gamma}_{i}$ and the stability of $\tilde{A}_{i}$ are opposite, $i=1,2,3,4$, so, by the generalized Poincare Bendixson Theorem, there must be at least one limit cycle which is contained in $\tilde{\Gamma}_{i}$ and is surrounding the critical point $\tilde{A}_{i}$, $i=1,2,3,4$. Now system (1.4) has at least 9 limit cycles and 8 homoclinic loops.

By an appropriate perturbation, the stability of $\tilde{A}_{i}$ changes according to the Hopf bifurcation, system (1.4) generates at least one limit cycle in the neighbourhood of $\tilde{A}_{i}$. By the same reason, there will be generated at least two limit cycles in the periphery of $\tilde{D}_{i}$. Under perturbation, the homoclinic loops $\tilde{\Gamma}_{i}, \bar{\Gamma}_{i}, i=1,2,3,4$, are broken, by homoclinic bifurcation, at least one limit cycle occurs in the inner neighbourhood of each $\tilde{\Gamma}_{i}, \bar{\Gamma}_{i}, i=1,2,3,4$. At the same time the limit cycles generated previously preserve their existence. Hence system (1.4) has now 29 limit cycles, and we have the following result.

THEOREM 1.1. There is a region $V$ in the parameter space $\{(\delta, \mu, r, k, n) \mid(\delta, \mu, r$, $\left.k, n) \in R^{5}\right\}$ such that system (1.4) has at least 29 limit cycles when $(\delta, \mu, r k, n) \in V$. The distribution of these limit cycles is shown as on Figure 3.

In Section 2, we shall outline how to sketch the graph of the curve $H(x, y)=h$; in Section 3, Hopf bifurcation and homoclinic bifurcation of system (1.4) are given; and in the last section we give a rigorous proof of our result. 


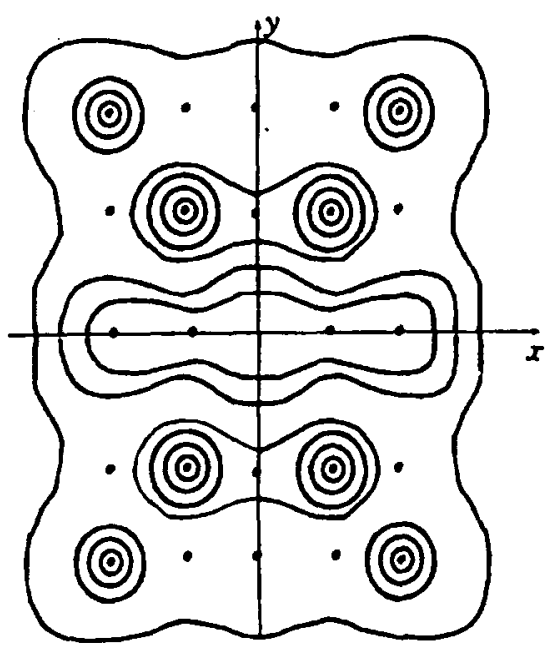

FIGURE 3.

\section{The graph of the curve $H(x, y)=h$}

It is easy to see that the vector field defined by system (1.2) is symmetric with respect to both $x$-axis and $y$-axis, so it suffices to consider the graph of $H(x, y)=h$ in the first quadrant.

Let $u=y^{2}-5, s=x^{2}-5 / 4$, then $H(x, y)=h$ becomes

$$
\begin{aligned}
& \Psi(u, s, h)=u^{3}-27 u+16 s^{3}-27 s-25 / 2-96 h=0, \\
& (s, u) \in \Omega=\{(s, u) \mid s \geq-5 / 4, u \geq-5\} .
\end{aligned}
$$

In order to sketch the graph of $H(x, y)=h$ in $(x, y)$ plane, it is sufficient to analyze the implicit function $u=u(s, h)$ satisfying $\Psi(u(s, h), s, h)=0$ in the region $u \geq-5$, $s \geq-5 / 4$. The implicit function $u=u(s, h)$ is closely related to the maximal and minimal values of function $\Psi=\Psi(u, s, h)$, which is regarded as a function of $u$ with parameters $s$ and $h$, and its value at $u=-5(\Psi(-5, s, h))$. By the methods of elementary calculus it is not difficult to calculate that the maximal and minimal values of $\Psi(u, s, h)$ are

$$
2 \varphi_{1}(s, h)=16 s^{3}-27 s+83 / 2-96 h, \quad s \geq-5 / 4,
$$

and

$$
2 \varphi_{2}(s, h)=16 s^{3}-27 s-133 / 2-96 h, \quad s \geq-5 / 4,
$$

respectively, and that

$$
\Psi(-5, s, h)=2 \varphi_{3}(s, h)=16 s^{3}-27 s-5 / 2-96 h, \quad s \geq-5 / 4 .
$$


It is straightforward to see that $\Psi(u, s, h) \geq 2 \varphi_{2}(s, h)$, for $u \geq-5$, and $\varphi_{2}(s, h)<$ $\varphi_{3}(s, h)<\varphi_{1}(s, h)$.

Equation (2.1) can also be regarded as a cubic algebraic equation of $u$ with parameters $s$ and $h$. Its discriminant is

$$
D(s, h)=\varphi_{1}(s, h) \varphi_{2}(s, h),
$$

where $\varphi_{1}(s, h)$ and $\varphi_{2}(s, h)$ are defined by (2.2) and (2.3). From (2.5) we know that

(1) if $\varphi_{2}(s, h)>0$ or $\varphi_{1}(s, h)<0$, then $D(s, h)>0$ and (2.1) has a unique real root;

(2) if $\varphi_{1}(s, h)=0$ or $\varphi_{2}(s, h)=0$, then $D(s, h)=0$ and (2.1) has two real roots, one of them a double root;

(3) if $\varphi_{2}(s, h)<0<\varphi_{1}(s, h)$, then $D(s, h)<0$ and (2.1) has three real roots.

Also $\varphi_{1}(s, h)=0, \varphi_{2}(s, h)=0$ and $\varphi_{3}(s, h)=0$ are cubic equations of $s$ with parameter $h$; their discriminants are

$$
\begin{gathered}
d_{1}(h)=9(h-7 / 24)(h-55 / 96), \quad d_{2}(h)=9(h+5 / 6)(h+53 / 96) \\
\text { and } d_{3}(h)=9(h-11 / 96)(h+1 / 6)
\end{gathered}
$$

respectively.

REMARK. Relations (2.2)-(2.4) can be rewritten in the following forms

$$
\begin{aligned}
& \varphi_{1}(s, h)=(s+5 / 4)\left(8 s^{2}-10 s-1\right)-48(h-11 / 24), \\
& \varphi_{2}(s, h)=(s+5 / 4)\left(8 s^{2}-10 s-1\right)-48(h+2 / 3),
\end{aligned}
$$

and

$$
\varphi_{3}(s, h)=(s+5 / 4)\left(8 s^{2}-10 s-1\right)-48 h .
$$

Since $d_{i}(h)=0, i=1,2,3$, and by the Remark we can obtain $h_{1}=-5 / 6$, $h_{2}=-2 / 3, h_{3}=-53 / 96, h_{4}=-1 / 6, h_{5}=0, h_{6}=11 / 96, h_{7}=7 / 24, h_{8}=11 / 24$, $h_{9}=55 / 96$, where $d_{i}(h), i=1,2,3$, are defined by (2.6). It is worth indicating that the critical points of system (1.2) correspond to the values $h_{i}, i=1,2, \ldots, 9$.

By analyzing the real roots of $\varphi_{i}(s, h)=0, i=1,2,3$, and hence of $\Psi(u, s, h)=0$, it is not difficult to sketch the graph of $H(x, y)=h$ in the first quadrant of the $(x, y)$ plane. We give only the analysis of the curve $H(x, y)=h$ for some values of $h$, say, $h \in(-\infty,-5 / 6), h=h_{1}, h \in(-5 / 6,-2 / 3)$, and $h=h_{2}$. The graph of $H(x, y)=h$ corresponding to the other values of $h$ can be obtained similarly. We list the results obtained in the table below.

1. $h \in(-\infty,-5 / 6)$.

In this case, $\varphi_{2}(s, h)>0$, for $s \geq-5 / 4$ (see Figure 4). Hence $D(s, h)>0$, for $s \geq-5 / 4, h<-5 / 6$ and $\Psi(u, s, h)>2 \varphi_{2}(s, h)$ for $u \geq-5$. The unique real root 


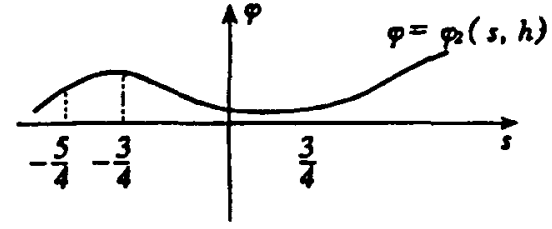

Figure 4.

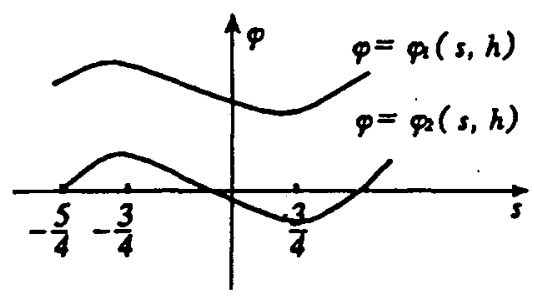

FIGURE 6.

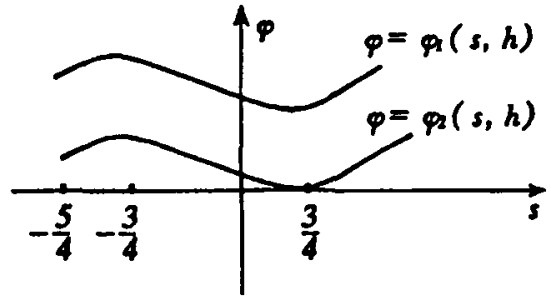

FIGURE 5.

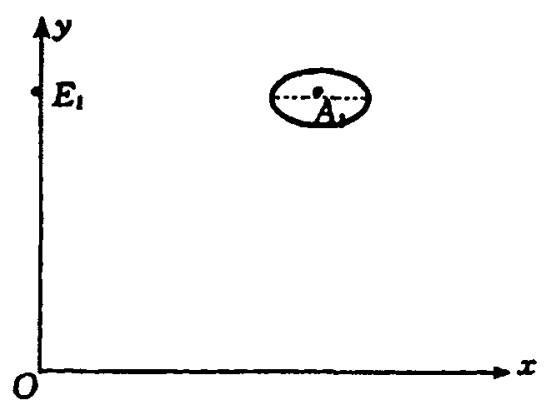

FIGURE 7.

of $\Psi(u, s, h)=0$ lies on the left of $u=-5$, in other words, $\Psi(u, s, h)>0$, for $(s, u) \in \Omega, h \in(-\infty,-5 / 6)$. So $H(x, y)=h$ has no real orbit in the $(x, y)$-plane.

2. $h=h_{1}=-5 / 6$.

In this case $\varphi_{2}(s, h) \geq 0$, for $s \geq-5 / 4$ and the unique real root of $\varphi_{2}(s, h)=0$ in the region $\{s \mid s \geq-5 / 4\}$ is $s_{1}=3 / 4$, which is a double root (see Figure 5).

$D\left(s, h_{1}\right)=\varphi_{2}\left(s, h_{1}\right) \varphi_{1}\left(s, h_{1}\right)>0$ if $s \in[-5 / 4,3 / 4) \cup(3 / 4,+\infty)$, and the unique real root of $\Psi(u, s, h)=0$ lies on the left of $u=-5$, so $\Psi(u, s, h)>0$ for $u \geq-5$, $s \in[-5 / 4,3 / 4) \cup(3 / 4, \infty)$. Therefore, $H(x, y)=h_{1}$ has no real orbit in the region $\{(x, y) \mid x \neq \pm \sqrt{2}\}$ of the $(x, y)$-plane.

As $s=s_{1}=3 / 4, \Psi\left(u, s_{1}, h_{1}\right)=(u+6)(u-3)^{2}$, and $u=3$ is the unique real root of $\Psi\left(u, s_{1}, h_{1}\right)=0$ in the region $\{u \mid u \geq-5\}$, in other words, $\left(s_{1}, u_{1}\right)=(3 / 4,3)$ is the unique real root of equation $\Psi\left(u, s, h_{1}\right)=0$ in the region $\Omega$. Consequently, the real orbits of $H(x, y)=h_{1}$ are critical points $A_{i}( \pm \sqrt{2}, \pm 2 \sqrt{2}), i=1,2,3,4$.

3. $h=h_{2}=-2 / 3$.

By the Remark, $\varphi_{2}\left(s, h_{2}\right)$ can be rewritten as

$$
\varphi_{2}\left(s, h_{2}\right)=8(s+5 / 4)(s-5 / 8+\sqrt{33} / 8)(s-5 / 8-\sqrt{33} / 8)
$$

so $\varphi_{2}\left(s, h_{2}\right)=0$ has three real roots: $s_{1}=-5 / 4, s_{2}=(5-\sqrt{33}) / 8$ and $s_{3}=(5+\sqrt{33}) / 8$ 


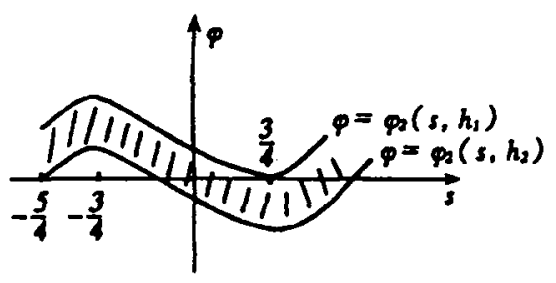

FigURE 8.

(see Figure 6). Since

$$
\Psi\left(u, s_{1}, h_{2}\right)=(u-3)^{2}(u+6)=0,
$$

the real root of $\Psi\left(u, s_{1}, h_{2}\right)=0$ in the region $\{u \mid u \geq-5\}$ is $u=3$, which is a double root, correspondingly, $H(x, y)=h_{2}$ has real orbits $E_{i}=\left(0,(-1)^{i-1} 2 \sqrt{2}\right), i=1,2$, which are critical points of system (1.2). While $s=s_{i}, i=2,3, \Psi\left(u, s_{i}, h_{2}\right)=$ $\Psi\left(u, s_{1}, h_{2}\right), i=2,3$, again $u=3$ is the real root of $\Psi\left(u, s_{i}, h_{2}\right)=0, i=2,3$, but their corresponding points in the $(x, y)$-plane are points

$$
\left( \pm \sqrt{\frac{15-\sqrt{33}}{8}}, \pm 2 \sqrt{2}\right), \quad\left( \pm \sqrt{\frac{15+\sqrt{33}}{8}}, \pm 2 \sqrt{2}\right) .
$$

As $s \in\left(-5 / 4, s_{1}\right) \cup\left(s_{2},+\infty\right), \varphi_{2}\left(s, h_{2}\right)>0$, and the unique real root of $\Psi\left(u, s, h_{2}\right)=0$ lies on the left of $u=-5$, so $\Psi\left(u, s, h_{2}\right)>0$ for $u \geq-5$, $s \in\left(-5 / 4, s_{1}\right) \cup\left(s_{2},+\infty\right)$. Therefore, $H(x, y)=h_{2}$ has no real orbit in the corresponding region of $(x, y)$-plane.

As $s \in\left(s_{1}, s_{2}\right), D\left(s, h_{2}\right)=\varphi_{2}\left(s, h_{2}\right) \varphi_{1}\left(s, h_{2}\right)<0$, equation $\Psi\left(u, s, h_{2}\right)=0$ has three real roots, one of them lies to the left of $u=-5$, the other lie to the right of $u=-5$. So $\Psi\left(u, s, h_{2}\right)=0$ has two real roots in the region $u \geq-5$, for $s \in\left(s_{1}, s_{2}\right)$. Therefore, $H(x, y)=h_{2}$ has two real orbits in the corresponding region of $(x, y)$ plane (see Figure 7).

4. $h \in\left(h_{1}, h_{2}\right), h_{1}=-5 / 6, h_{2}=-2 / 3$.

In this case $\varphi_{2}\left(s, h_{2}\right)<\varphi_{2}(s, h)<\varphi_{2}\left(s, h_{1}\right)$, the curve $\varphi=\varphi_{2}(s, h)$ always lies between the curves $\varphi=\varphi_{2}\left(s, h_{2}\right)$ and $\varphi_{2}\left(s, h_{1}\right)$ for $h \in(-5 / 6,-2 / 3)$ (see the shaded region in Figure 8). So $\varphi_{2}(s, h)=0$ always has two real roots $s_{1}(h)$ and $s_{2}(h)$ in the region $s \geq-5 / 4$, we assume that $s_{1}(h)<s_{2}(h)$. Since $D\left(s_{i}(h), h\right)=0$, thus $\Psi\left(u, s_{i}(h), h\right)=0$ has two real roots $u=3$ and $u=-6$ for $i=1,2$, where $u=3$ is a double root and $u=-6$ is less than $u=-5$. Correspondingly, the real orbits of $H(x, y)=h$ are points $\left(\sqrt{s_{i}(h)+5 / 4}, 2 \sqrt{2}\right),(i=1,2)$.

Also $\varphi_{1}(s, h) \geq \varphi_{1}(3 / 4, h)=14-48 h>0$, if $s \in\left(s_{1}(h), s_{2}(h)\right)$, then $\varphi_{2}(s, h)<$ $0<\varphi_{1}(s, h)$, hence $D(s, h)<0$. Therefore, $\Psi(u, s, h)=0$ has three real roots, $u=$ 


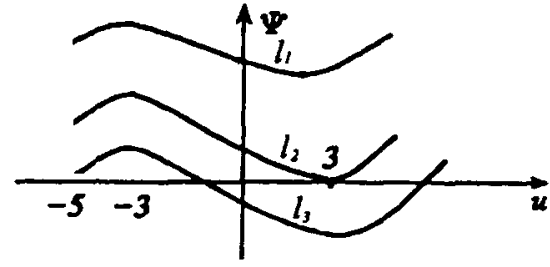

FIGURE 9.

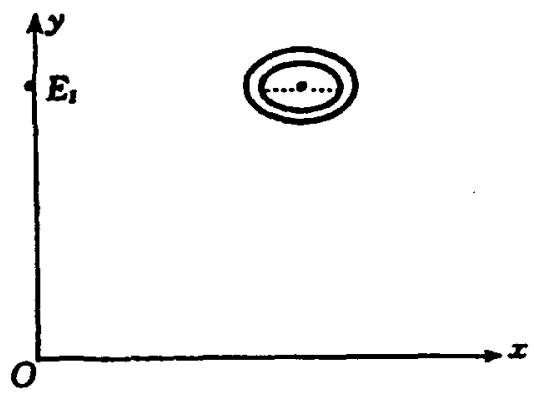

FIGURE 10.

$u_{i}(s, h), i=1,2,3$. Assume that $u_{1}(s, h)<u_{2}(s, h)<u_{3}(s, h)$, then $u_{1}(s, h)<-5$, $-3<u_{2}(s, h)<3<u_{3}(s, h)$. By the continuity of $u(s, h)$, when $s=s_{i}(h)$, $u_{2}\left(s_{i}(h), h\right)=u_{3}\left(s_{i}(h), h\right), i=1,2$. So the corresponding real orbits of $H(x, y)=h$ are closed orbits which surround critical point $A_{j}, j=1,2,3,4$, as $s \in\left[s_{1}(h), s_{2}(h)\right]$.

If $s \in\left[-5 / 4, s_{1}(h)\right) \cup\left(s_{2}(h),+\infty\right)$, then $\varphi_{2}(s, h)>0$ and so $D(s, h)>0$. In this case, the unique real root of $\Psi(u, s, h)=0$ lies on the left of $u=-5$. Therefore, as we have analyzed above, $H(x, y)=h$ has no real orbit in the corresponding region of $(x, y)$ plane.

Summing up, if $h \in(-5 / 6,-2 / 3)$, the orbits of $H(x, y)=h$ are closed orbits which surround critical points $A_{j}, j=1,2,3,4$.

Figure 9 shows the graph of $\Psi=\Psi(u, s, h)$ corresponding to (i) $\varphi_{2}(s, h)>0\left(l_{1}\right)$; (ii) $\varphi_{2}(s, h)=0\left(l_{2}\right)$; (iii) $\varphi_{2}(s, h)<0$ and $\varphi_{3}(s, h)>0\left(l_{3}\right)$, respectively. While Figure 10 shows the orbits of $H(x, y)=h$ in the first quadrant of $(x, y)$-plane for $h \in[-5 / 6,-2 / 3]$.

The graph of $H(x, y)=h$ in the first quadrant of $(x, y)$-plane for other values of parameter $h$ are shown in Table 1.

\section{Hopf bifurcation and homoclinic bifurcation}

Now we consider the Hopf bifurcation of (1.4) at $\tilde{A}_{1}$. Let $\bar{x}=x-\alpha, \bar{y}=y-2 \sqrt{2}$, where $(\alpha, 2 \sqrt{2})$ is the coordinate of $\tilde{A}_{1}$, then (1.4) becomes

$$
\begin{aligned}
\mathrm{d} \bar{x} / \mathrm{d} t= & 6 \bar{y}+25 \sqrt{2} \bar{y}^{2} / 4+35 \bar{y}^{3} / 8+5 \sqrt{2} \bar{y}^{4} / 8+\bar{y}^{5} / 16, \\
\mathrm{~d} y / \mathrm{d} t= & \bar{b}_{01} \bar{x}+\bar{b}_{02} \bar{y}+\bar{b}_{1} \bar{x}^{2}+\bar{b}_{2} \bar{x} \bar{y}+\bar{b}_{3} \bar{y}^{2}+\bar{b}_{4} \bar{x}^{3}+\bar{b}_{5} \bar{x}^{2} \bar{y}+\bar{b}_{6} \bar{x} \bar{y}^{2} \\
& +\bar{b}_{7} \bar{y}^{3}+\bar{b}_{8} \bar{x}^{4}+\bar{b}_{9} \bar{x}^{3} \bar{y}+\bar{b}_{10} \bar{x}^{2} \bar{y}^{2}+\bar{b}_{11} \bar{x} \bar{y}^{3}+\bar{b}_{12} \bar{y}^{4}+\bar{b}_{13} \bar{x}^{5} \\
& +\bar{b}_{14} \bar{x}^{4} \bar{y}+\bar{b}_{16} \bar{x}^{2} \bar{y}^{3}+\bar{b}_{18} \bar{y}^{5},
\end{aligned}
$$


[9]

The number of limit cycles

45

TABLE 1. The graph for $H(x, y)=h$ in the first quadrant corresponding to different values of parameter $h$

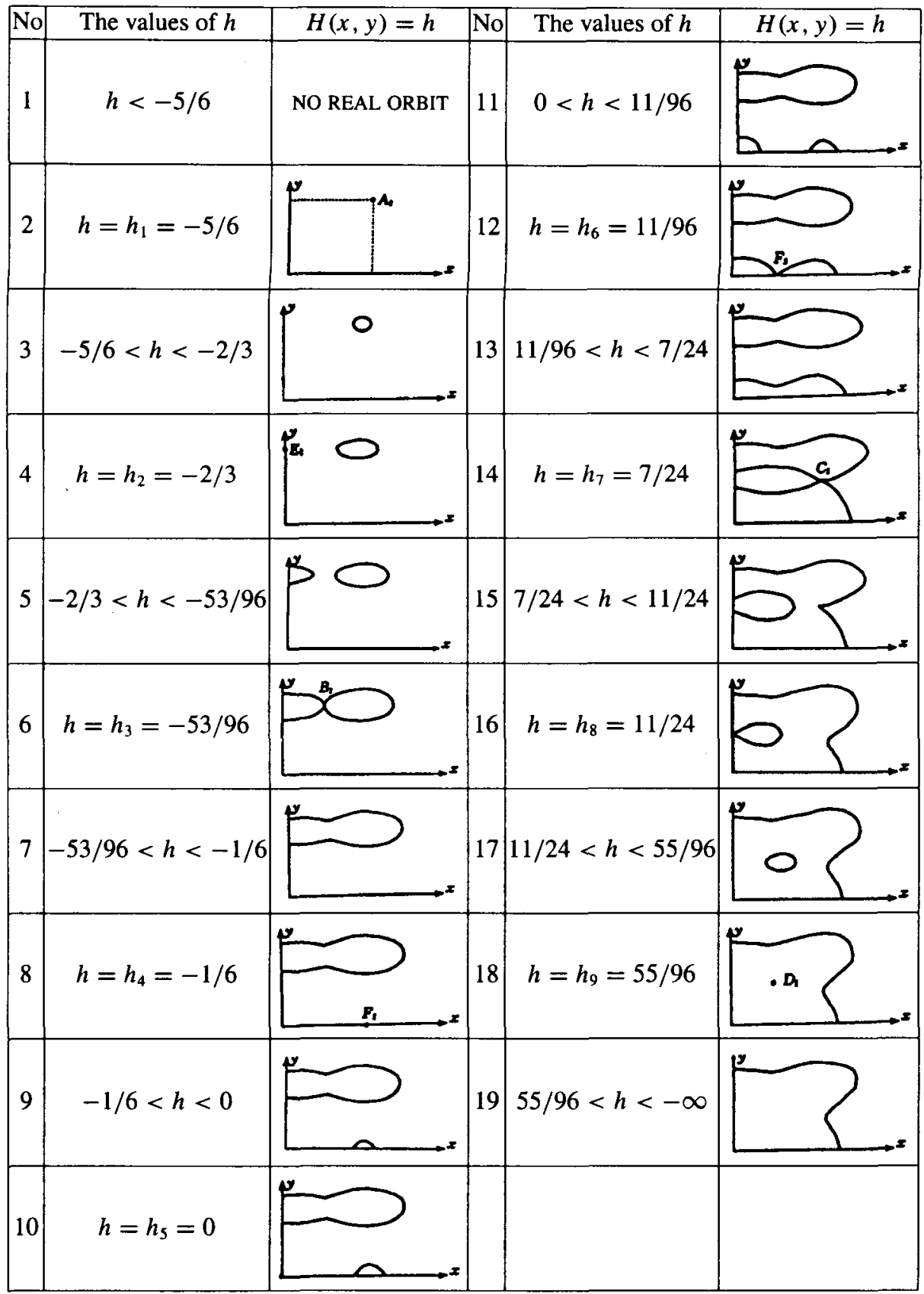

https://doi.org/10.1017/S1446788700008466 Published online by Cambridge University Press 
where $\bar{b}_{02}=\varepsilon\left(\delta+\alpha^{2} \mu+24 r+\alpha^{4} k+24 \alpha^{2} n+320\right)$ and $\bar{b}_{01}, \bar{b}_{1}, \bar{b}_{2}, \ldots, \bar{b}_{14}, \bar{b}_{16}, \bar{b}_{18}$ are constants which are relative to the parameters $\mu, r, k, n$ and $\varepsilon$. The necessary condition for the origin being a fine focus or a center of system (3.1) is $\bar{b}_{02}=0$. Because $0<\varepsilon \ll 1$, thus

$$
\delta+\alpha^{2} \mu+24 r+\alpha^{4} k+24 \alpha^{2} n+320=0 .
$$

Since $\left|\alpha^{2}-2\right| \ll 1$, we may assume that $\alpha=\sqrt{2}+b \varepsilon+o(\varepsilon)$. We notice that $(\alpha, 2 \sqrt{2})$ is a critical point of system (1.4), so by (3.2), we have $b=-16 \sqrt{2} r / 3-$ $32 \sqrt{2} n / 3-256 \sqrt{2} / 3$. Hence the first approximation of the first order Hopf bifurcation at $\tilde{A}_{1}(\alpha, 2 \sqrt{2})$ is

$$
L_{H B}^{(1)}: \delta+2 \mu+24 r+4 k+48 n+320=0 .
$$

Let $\bar{x}=\zeta / c, \bar{y}=\eta / \sigma$ and $\mathrm{d} t / \mathrm{d} \tau=1 / \sigma$, where $c=6-\varepsilon(8 \mu+800 r / 3+32 k+$ $1792 n / 3+12800 / 3)+o(\varepsilon), \sigma^{2}=6 c$ (or $\sigma=\sqrt{6} c$ ), then we have

$$
\left\{\begin{aligned}
\frac{\mathrm{d} \zeta}{\mathrm{d} \tau}= & \eta+\frac{25 \sqrt{2}}{24 \sigma} \eta^{2}+\frac{35}{48 \sigma^{2}} \eta^{3}+\frac{5 \sqrt{2}}{48 \sigma^{3}} \eta^{4}+\frac{1}{96 \sigma^{4}} \eta^{5} \\
\frac{\mathrm{d} \eta}{\mathrm{d} \tau}= & -\zeta+b_{1} \zeta^{2}+b_{2} \zeta \eta+b_{3} \eta^{2}+b_{4} \zeta^{3}+b_{5} \zeta^{2} \eta+b_{6} \zeta \eta^{2}+b_{7} \eta^{3} \\
& +b_{8} \zeta^{4}+b_{9} \zeta^{3} \eta+b_{10} \zeta^{2} \eta^{2}+b_{11} \zeta \eta^{3}+b_{12} \eta^{4}+b_{13} \zeta^{5} \\
& +b_{14} \zeta^{4} \eta+b_{16} \zeta^{2} \eta^{3}+b_{18} \eta^{5}
\end{aligned}\right.
$$

where $b_{i}(i=1,2, \ldots, 14,16,18)$ are constants which are relative to the parameters $\mu, r, k, n$ and $\varepsilon$.

By the formulae of focal quantities to the polynomial system (Zhang [8]), we have $W_{1}=\varepsilon(11 \mu+33 r+32 k+330 n+640) / 1296+o(\varepsilon)$. Therefore, the first approximation of the second order Hopf bifurcation at $\tilde{A}_{1}(\alpha, 2 \sqrt{2})$ is

$$
L_{H B}^{(2)}:\left\{\begin{array}{r}
\delta+2 \mu+24 r+4 k+48 n+320=0, \\
11 \mu+33 r+32 k+330 n+640=0 .
\end{array}\right.
$$

The symmetry of the vector field (1.2) with respect to both $x$-axis and $y$-axis implies that the first approximation of the first and the second orders Hopf bifurcations at $\tilde{A}_{2}\left(\alpha_{2}, 2 \sqrt{2}\right), \tilde{A}_{3}\left(\alpha_{3},-2 \sqrt{2}\right)$ and $\tilde{A}_{4}\left(\alpha_{4},-2 \sqrt{2}\right)$ are the same as that at $\tilde{A}_{1}(\alpha, 2 \sqrt{2})$.

By the same way we can obtain the first approximations of the first and second orders Hopf bifurcations at $\tilde{D}_{i}\left(p_{i},(-1)^{[(i-1) / 2]} \sqrt{2}\right), i=1,2,3,4$, they are

$$
L_{H B}^{(1)}: \delta+\mu / 2+6 r+k / 4+3 n+20=0
$$


and

$$
L_{H B}^{(2)}:\left\{\begin{array}{r}
\delta+\mu / 2+6 r+k / 4+3 n+20=0, \\
80-15 n+4 k-6 r-2 \mu=0,
\end{array}\right.
$$

respectively, where $[(i-1) / 2]$ is the integer part of $(i-1) / 2$.

As we have mentioned above (Chow [2]), when $\varepsilon$ is very small and if

$$
M(h)=\oint_{\Gamma_{h}}\left(\delta y+\mu x^{2} y+r y^{3}+k x^{4} y+n x^{2} y^{3}+y^{5}\right) \mathrm{d} x=0
$$

holds, then there are limit cycles of (1.4) in the neighbourhood of $\Gamma_{h}$, where $\Gamma_{h}$ is a compact component of $H(x, y)=h$ and $H(x, y)$ is defined in (1.3). If $\Gamma_{h}$ is a homoclinic loop of (1.2) which passes through a saddle point, then the validity of (3.8) also shows that near $\Gamma_{h}$ there exists a separatrix loop of (1.4) passing through a saddle point. Thus $M(h)$ is the first approximation $L_{H L B}$ of homoclinic loop bifurcation of (1.4).

Let $h=h_{3}=-53 / 96$, then $\Gamma_{h_{3}}$ has four branches, each branch is a singular closed orbit which passes through a saddle point $B_{i}$ and surrounds only $A_{i}(i=1,2,3,4)$. We first consider a branch of $\Gamma_{h_{3}}$ which passes through $B_{1}$ and surrounds $A_{1}$. The first approximation of homoclinic bifurcation surface to (1.4) is

$$
L_{H L B}: \oint_{\Gamma_{h_{3}}^{(1)}}\left(\delta y+\mu x^{2} y+r y^{3}+k x^{4} y+n x^{2} y^{3}+y^{5}\right) \mathrm{d} x=0 .
$$

It passes through saddle point $\tilde{B}_{1}$ and surrounds $\tilde{A}_{1}$, where

$$
\Gamma_{h_{3}}: \frac{x^{2}}{2}+\frac{y^{2}}{2}-\frac{5 x^{4}}{8}-\frac{5 y^{4}}{32}+\frac{x^{6}}{6}+\frac{y^{6}}{96}=-\frac{53}{96}, \quad \frac{\sqrt{2}}{2} \leq x \leq \frac{\sqrt{11}}{2},
$$

and $\Gamma_{h_{3}}^{(1)}$ is a branch of $\Gamma_{h_{3}}$ which passes through $B_{1}$ and surrounds $A_{1}$.

By calculation, (3.9) can be expressed as follows

$$
L_{H L B}: 0.3825 \delta+0.6501 \mu+8.9927 r+1.2152 k+15.2548 n+118.7860=0 .
$$

Since the orbits of (1.2) are symmetric with respect to both $x$-axis and $y$-axis (see Figure 1), the first approximation of the homoclinic bifurcation surface which passes through saddle point $\tilde{B}_{i}$ and surrounds $\tilde{A}_{i}, i=2,3,4$ are also (3.10).

If $h=h_{8}=11 / 24$, then $\Gamma_{h_{8}}$ has four branches, the first branch passes through saddle point $E_{3}(0, \sqrt{2})$ and surrounds $D_{1}$; the second one passes through $E_{3}$ and surrounds $D_{2}$; the third one passes through $E_{4}(0,-\sqrt{2})$ and surrounds $D_{3}$; and the last one passes through $E_{4}$ and surrounds $D_{4}$. Similarly, the first approximations of the homoclinic bifurcation surfaces of the other three branches are

(3.11) $L_{H L B}:-0.5825 \delta-0.2571 \mu-3.4490 r-0.1648 k-1.5213 n-12.1861=0$. 


\section{Proof of Theorem 1.1}

Based on a paragraph in Section 3, we can now sketch the first approximation of Hopf bifurcation surfaces and homoclinic bifurcation surfaces (3.3), (3.7), (3.10) and (3.11) in the parameter space $\left\{(\delta, \mu, r, k, n) \mid(\delta, \mu, r, k, n) \in R^{5}\right\}$, and find their intersection point $(\bar{\delta}, \bar{\mu}, \bar{r}, \bar{k}, \bar{n})$. In fact, from the linear algebraic equations

$$
\begin{aligned}
\delta+2 \mu+24 r+4 k+12 n+320 & =0, \\
\delta+\mu / 2+6 r+k / 4+3 n+20 & =0, \\
-2 \mu-6 r+4 k-15 n+80 & =0, \\
0.3825 \delta+0.6501 \mu+8.9927 r+1.2152 k+15.2548 n+118.7860 & =0, \\
-0.5825 \delta-0.2571 \mu-3.4490 r-0.1648 k-1.5213 n-12.1861 & =0,
\end{aligned}
$$

we have $\bar{\delta} \doteq 49.5065, \bar{\mu} \doteq 17.8431, \bar{r} \doteq-11.5151, \bar{k} \doteq-30.4935, \bar{n} \doteq-0.5713$.

LEMMA 1. If $(\delta, \mu, r, k, n)=(\bar{\delta}, \bar{\mu}, \bar{r}, \bar{k}, \bar{n})$ then there are limit cycles of system (1.4) which surround single critical points $\tilde{A}_{i}, i=1,2,3,4$, respectively (see Figure 2).

PROOF. When $(\delta, \mu, r, k, n)=(\bar{\delta}, \bar{\mu}, \bar{r}, \bar{k}, \bar{n}), W_{1} \doteq-0.5463 \varepsilon+o(\varepsilon)$. By the formulae of focal quantities (Zhang [8]) it is easy to see that $\tilde{A}_{i}(i=1,2,3,4)$ is an unstable first order fine focus. However, when $(\delta, \mu, r, k, n)=(\bar{\delta}, \bar{\mu}, \bar{r}, \bar{k}, \bar{n})$, the separatrix cycle which passes through $\tilde{B}_{i}$ and surrounds $\tilde{A}_{i}(i=1,2,3,4)$ is inner unstable; this is due to the fact that $M\left(h_{3}\right)=0$ and $\div\left.\left(P_{\varepsilon}, Q_{\varepsilon}\right)\right|_{\tilde{B}_{i}}=\left(\partial P_{\varepsilon} / \partial x+\right.$ $\left.\partial Q_{\varepsilon} / \partial y\right)\left.\right|_{\tilde{B}_{i}}=\varepsilon(\bar{\delta}+\bar{\mu} / 2+24 \bar{r}+\bar{k} / 4+12 \bar{n}+320) \doteq 87.5866 \varepsilon>0$. Hence by the generalized Poincare-Bendixson Theorem (Ye [7]), there is at least one limit cycle surrounding $\tilde{A}_{i}$ and lying inside the separatrix loop which passes $\tilde{B}_{i}(i=1,2,3,4)$ (see Figure 2).

LEMMA 2. If $(\delta, \mu, r, k, n)=(\bar{\delta}, \bar{\mu}, \bar{r}, \bar{k}, \bar{n})$, then there exists a $h_{10} \in(7 / 24,11 / 24)$ such that $\Gamma_{h_{10}}: x^{2} / 2+y^{2} / 2-5 x^{4} / 8-5 y^{4} / 32+x^{6} / 6+y^{6} / 96=h_{10}$ has two connected components $\Gamma_{h_{10}}^{(1)}$ and $\Gamma_{h_{10}}^{(2)}$ which are closed orbits of (1.2), and

$$
M\left(h_{10}\right)=\oint_{\Gamma_{h_{10}}^{(i)}}\left(\bar{\delta} y+\bar{\mu} x^{2} y+\bar{r} y^{3}+\bar{k} x^{4} y+\bar{n} x^{2} y^{3}+y^{5}\right) \mathrm{d} x=0, \quad i=1,2 .
$$

$\Gamma_{h_{10}}^{(1)}$ simultaneously surrounds singular points $D_{1}, D_{2}$ and $E_{3}$, and $\Gamma_{h_{10}}^{(2)}$ simultaneously surrounds singular points $D_{3}, D_{4}$ and $E_{4}$. Namely, there is a limit cycle of (1.4) near each connected component of $\Gamma_{h_{10}}$. 
PROOF. If $(\delta, \mu, r, k, n)=(\bar{\delta}, \bar{\mu}, \bar{r}, \bar{k}, \bar{n})$, let $h=\bar{h}=7 / 16$. By calculation, $M(\bar{h}) \doteq-0.0102$, in which

$$
\Gamma_{\bar{h}}: \frac{x^{2}}{2}+\frac{y^{2}}{2}-\frac{5 x^{4}}{8}-\frac{5 y^{4}}{32}+\frac{x^{6}}{6}+\frac{y^{6}}{96}=\frac{7}{16}, \quad-1.1097 \leq x \leq 1.1097 .
$$

Again let $h=1 / 3$, then $M(1 / 3) \doteq 1.4198>0$, in which

$$
\cdot \Gamma_{1 / 3}: \frac{x^{2}}{2}+\frac{y^{2}}{2}-\frac{5 x^{4}}{8}-\frac{5 y^{4}}{32}+\frac{x^{6}}{6}+\frac{y^{6}}{96}=\frac{1}{3}, \quad-1.2790 \leq x \leq 1.2790 .
$$

Since $M(h)=\oint_{\Gamma_{h}}\left(\bar{\delta} y+\bar{\mu} x^{2} y+\bar{r} y^{3}+\bar{k} x^{4} y+\bar{n} x^{2} y^{3}+y^{5}\right) \mathrm{d} x$ is continuous with respect to $h$ (Chow [2]), there exists $h_{10} \in(1 / 3,7 / 16) \subset(7 / 24,11 / 24)$ such that $\Gamma_{h_{10}}: x^{2} / 2+y^{2} / 2-5 x^{4} / 8-5 y^{4} / 32+x^{6} / 6+y^{6} / 96=h_{10}$ consists of two closed orbits of (1.2) and

$$
M\left(h_{10}\right)=\oint_{\Gamma_{h_{10}}^{(i)}}\left(\bar{\delta} y+\bar{\mu} x^{2} y+\bar{r} y^{3}+\bar{k} x^{4} y+\bar{n} x^{2} y^{3}+y^{5}\right) \mathrm{d} x=0, \quad i=1,2,
$$

where $\Gamma_{h_{10}}^{(1)}$ is a branch of $\Gamma_{h_{10}}$ which simultaneously surrounds singular points $D_{1}, D_{2}$ and $E_{3}$; and $\Gamma_{h_{10}}^{(2)}$ is another branch of $\Gamma_{h_{10}}$ which surrounds singular points $D_{3}, D_{4}$ and $E_{4}$. The proof is complete.

LEMMA 3. If $(\delta, \mu, r, k, n)=(\bar{\delta}, \bar{\mu}, \bar{r}, \bar{k}, \bar{n})$, then there exist $h_{11} \in(11 / 96,39 / 192)$ and $h_{12} \in(39 / 192,7 / 24)$ such that $\Gamma_{h_{i}}: x^{2} / 2+y^{2} / 2-5 x^{4} / 8-5 y^{4} / 32+x^{6} / 6+$ $y^{6} / 96=h_{i}, i=11,12$, has a connected component $\Gamma_{h_{i}}^{\prime}(i=11,12)$, which is a closed orbit of (1.2), and

$$
M\left(h_{i}\right)=\oint_{\Gamma_{h_{i}}^{\prime}}\left(\bar{\delta} y+\bar{\mu} x^{2} y+\bar{r} y^{3}+\bar{k} x^{4} y+\bar{n} x^{2} y^{3}+y^{5}\right) \mathrm{d} x=0, \quad i=11,12,
$$

where $\Gamma_{h_{11}}^{\prime} \subset \Gamma_{h_{12}}^{\prime}$ and $\Gamma_{h_{11}}^{\prime}$ simultaneously surrounds singular points $O, F_{1}, F_{2}, F_{3}$ and $F_{4}$. It means that there are two limit cycles of (1.4), which lie in the neighbourhoods of $\Gamma_{h_{11}}^{\prime}$ and $\Gamma_{h_{12}}^{\prime}$ respectively.

PROOF. If $(\delta, \mu, r, k, n)=(\bar{\delta}, \bar{\mu}, \bar{r}, \bar{k}, \bar{n})$, let $h=h_{6}=11 / 96$, then $\Gamma_{h_{6}}$ is a singular closed orbit passing through saddle points $F_{3}$ and $F_{4}$ (see Figure 1). By calculation, we obtain

$$
M\left(h_{6}\right)=\oint_{\Gamma_{h_{6}}}\left(\bar{\delta} y+\bar{\mu} x^{2} y+\bar{r} y^{3}+\bar{k} x^{4} y+\bar{n} x^{2} y^{3}+y^{5}\right) \mathrm{d} x \doteq-0.8815
$$


where $\Gamma_{h_{6}}: x^{2} / 2+y^{2} / 2-5 x^{4} / 8-5 y^{4} / 32+x^{6} / 6+y^{6} / 96=11 / 96,-\sqrt{11} / 2 \leq$ $x \leq \sqrt{11} / 2$. Again let $h=h^{*}=8959 / 30720<h_{7}=7 / 24$, then $\Gamma_{h^{*}}: x^{2} / 2+$ $y^{2} / 2-5 x^{4} / 8-5 y^{4} / 32+x^{6} / 6+y^{6} / 96=8959 / 30720,-1.7116 \leq x \leq 1.7116$, has a branch of closed orbit $\Gamma_{h^{*}}^{\prime}$ to system (1.2), which simultaneously surrounds singular points $O, F_{1}, F_{2}, F_{3}$ and $F_{4}$. By calculation, it follows that

$$
M\left(h^{*}\right)=\oint_{\Gamma_{h^{*}}}\left(\bar{\delta} y+\bar{\mu} x^{2} y+\bar{r} y^{3}+\bar{k} x^{4} y+\bar{n} x^{2} y^{3}+y^{5}\right) \mathrm{d} x \doteq-0.0185 .
$$

At last let $h=\tilde{h}=39 / 192,11 / 96<39 / 192<8959 / 30720$, then there is a closed orbit $\Gamma_{\tilde{h}}^{\prime}$ of system (1.2) which is a branch of $\Gamma_{\bar{h}}$ lying between $\Gamma_{h_{6}}$ and $\Gamma_{h^{*}}^{\prime}$. Of course $\Gamma_{\bar{h}}^{\prime}$ simultaneously surrounds singular points $O, F_{1}, F_{2}, F_{3}$ and $F_{4}$. By calculation, we have

$$
M(\tilde{h})=\oint_{\Gamma_{\bar{h}}^{\prime}}\left(\bar{\delta} y+\bar{\mu} x^{2} y+\bar{r} y^{3}+\bar{k} x^{4} y+\bar{n} x^{2} y^{3}+y^{5}\right) \mathrm{d} x \doteq 17.2495
$$

Therefore, there exist $h_{11} \in(11 / 96,39 / 192)$ and $h_{12} \in(39 / 192,8959 / 30720) \subset$ $(39 / 192,7 / 24)$ such that $\Gamma_{h_{i}}: x^{2} / 2+y^{2} / 2-5 x^{4} / 8-5 y^{4} / 32+x^{6} / 6+y^{6} / 96=h_{i}$ has a branch of closed orbit $\Gamma_{h_{i}}^{\prime}$ to system (1.2) $(i=11,12)$, and

$$
M\left(h_{i}\right)=\oint_{\Gamma_{h_{i}}^{\prime}}\left(\bar{\delta} y+\bar{\mu} x^{2} y+\bar{r} y^{3}+\bar{k} x^{4} y+\bar{n} x^{2} y^{3}+y^{5}\right) \mathrm{d} x=0, \quad i=11,12,
$$

where $\Gamma_{h_{11}}^{\prime} \subset \Gamma_{h_{12}}^{\prime}$ and $\Gamma_{h_{11}}^{\prime}$ simultaneously surrounds singular points $O, F_{1}, F_{2}, F_{3}$ and $F_{4}$.

It is shown that there are two limit cycles of (1.4) which lie in the neighbourhoods of $\Gamma_{h_{11}}^{\prime}$ and $\Gamma_{h_{12}}^{\prime}$ respectively.

LEMMA 4. If $(\delta, \mu, r, k, n)=(\bar{\delta}, \bar{\mu}, \bar{r}, \bar{k}, \bar{n})$, then there exists $h_{13} \in(7 / 24,1) \subset$ $(7 / 24,+\infty)$, such that $\Gamma_{h_{13}}: x^{2} / 2+y^{2} / 2-5 x^{4} / 8-5 y^{4} / 32+x^{6} / 6+y^{6} / 96=h_{13}$ has a connected component $\Gamma_{h_{13}}^{\prime}$, which is a closed orbit of (1.2) simultaneously surrounding all 25 singular points and

$$
M\left(h_{13}\right)=\oint_{\Gamma_{h_{13}}^{\prime}}\left(\bar{\delta} y+\bar{\mu} x^{2} y+\bar{r} y^{3}+\bar{k} x^{4} y+\bar{n} x^{2} y^{3}+y^{5}\right) \mathrm{d} x=0 .
$$

This means that there is a limit cycle of (1.4) which lies in the neighbourhood of $\Gamma_{h_{13}}^{\prime}$.

Proof. If $(\delta, \mu, r, k, n)=(\bar{\delta}, \bar{\mu}, \bar{r}, \bar{k}, \bar{n})$, let $h=h_{14}=1 / 3$. Then $\Gamma_{h_{14}}$ has a connected component $\Gamma_{h_{14}}^{\prime}: x^{2} / 2+y^{2} / 2-5 x^{4} / 8-5 y^{4} / 32+x^{6} / 6+y^{6} / 96=1 / 3$, 
$-1.8404 \leq x \leq 1.8404$, which is a closed orbit of (1.2) simultaneously surrounding all 25 singular points. By calculation we have

$$
M\left(h_{14}\right)=\oint_{\Gamma_{h_{14}}^{\prime}}\left(\bar{\delta} y+\bar{\mu} x^{2} y+\bar{r} y^{3}+\bar{k} x^{4} y+\bar{n} x^{2} y^{3}+y^{5}\right) \mathrm{d} x \doteq 69.0355
$$

Similarly, let $h=h_{15}=1$, then $\Gamma_{h_{15}}$ is a closed orbit of (1.2) and surrounds all 25 singular points, and by calculation, we have

$$
M\left(h_{15}\right)=\oint_{\Gamma_{h_{15}}}\left(\bar{\delta} y+\bar{\mu} x^{2} y+\bar{r} y^{3}+\bar{k} x^{4} y+\bar{n} x^{2} y^{3}+y^{5}\right) \mathrm{d} x \doteq-151.8140 .
$$

Therefore, there exists $h_{13} \in(1 / 3,1) \subset(7 / 24,+\infty)$ such that $\Gamma_{h_{13}}: x^{2} / 2+y^{2} / 2-$ $5 x^{4} / 8-5 y^{4} / 32+x^{6} / 6+y^{6} / 96=h_{13}$ has a connected component $\Gamma_{h_{13}}^{\prime}$ which is a closed orbit of (1.2) simultaneously surrounding all 25 singular points, and

$$
M\left(h_{13}\right)=\oint_{\Gamma_{h_{13}^{\prime}}}\left(\bar{\delta} y+\bar{\mu} x^{2} y+\bar{r} y^{3}+\bar{k} x^{4} y+\bar{n} x^{2} y^{3}+y^{5}\right) \mathrm{d} x=0 .
$$

It is shown that there is a limit cycle of (1.4), which lies in the neighbourhood of $\Gamma_{h_{13}}^{\prime}$.

THEOREM 4.1. If $(\delta, \mu, r, k, n)=(\bar{\delta}, \bar{\mu}, \bar{r}, \bar{k}, \bar{n})$, then system (1.4) has 9 limit cycles and 8 homoclinic cycles (see Figure 2 ).

Theorem 4.1 follows from Lemma 1-Lemma 4 immediately.

THEOREM 4.2. If $(\delta, \mu, r, k, n)=(\bar{\delta}, \bar{\mu}, \bar{r}, \bar{k}, \bar{n})$, then $\tilde{D}_{i}, i=1,2,3,4$, are stable second order fine foci of (1.4).

Proof. In this case, $\bar{W}_{1}=0, \bar{W}_{2} \doteq-0.1381<0$. Since $\mathrm{d} t / \mathrm{d} \tau=-1 / \sigma<0$, singular point $\tilde{D}_{1}$ of (1.4) is a stable second order fine focus. By the symmetry, the other singular points $\tilde{D}_{i}, i=2,3,4$ of (1.4) are also stable second order fine foci.

THEOREM 4.3. If $(\delta, \mu, r, k, n)=(\bar{\delta}, \bar{\mu}, \bar{r}, \bar{k}, \bar{n})$, then $\tilde{A}_{i}, i=1,2,3,4$ are unstable first order fine foci of (1.4).

Proof. In this case $W_{1} \doteq-0.5463<0$. Since $\mathrm{d} t / \mathrm{d} \tau=1 / \sigma>0$, a singular point $\tilde{A}_{1}$ of (1.4) is an unstable first order fine focus.

By the symmetry, the other singular points $\tilde{A}_{i}, i=2,3,4$ of (1.4) are also unstable first order fine foci. 
PROOF OF THEOREM 1.1. By the continuity of limit cycles with respect to the variation of parameters (Ye [7]), we know that there is a neighbourhood $\Sigma$ of $(\bar{\delta}, \bar{\mu}, \bar{r}, \bar{k}, \bar{n})$, such that when $(\delta, \mu, r, k, n) \in \Sigma$ system (1.4) still has 9 limit cycles, (see for example Figure 2). Let $\Sigma_{1}=\Sigma \cap\{(\delta, \mu, r, k, n) \mid 0<-(\delta+2 \mu+24 r+4 k+48 n+320) \ll$ $1, \delta+\mu / 2+6 r+k / 4+3 n+20=0,0<80-15 n+4 k-6 r-2 \mu \ll 1\}$, then, when $(\delta, \mu, r, k, n) \in \Sigma_{1}, \tilde{A}_{i}, i=1,2,3,4$, become stable rough foci, so an unstable limit cycle with small amplitude appears near each of $\tilde{A}_{i}(i=1,2,3,4)$. At the same time $\tilde{D}_{i}, i=1,2,3,4$, become unstable first order fine foci, so, a stable limit cycle with small amplitude also appears near each of $\tilde{D}_{i}(i=1,2,3,4)$.

Let $\Sigma_{2}=\Sigma_{1} \cap\{(\delta, \mu, r, k, n) \mid 0<-(\delta+\mu / 2+6 r+k / 4+3 n+20) \ll 1\}$, when $(\delta, \mu, r, k, n) \in \Sigma_{2}, \tilde{D}_{i}, i=1,2,3,4$, become stable rough foci, so an unstable limit cycle with small amplitude appears near each of $\tilde{D}_{i}(i=1,2,3,4)$.

Let $\Sigma_{3}=\Sigma_{2} \cap\{(\delta, \mu, r, k, n) \mid(3.10)$ and (3.11) hold $\}$, then when $(\delta, \mu, r, k, n)$ $\in \Sigma_{3}$, system (1.4) has 21 limit cycles and 8 homoclinic cycles. Denote $\Gamma_{h_{3}}^{i}$ the homoclinic cycle which surrounds $\tilde{A}_{i}$ and passes through saddle point $\tilde{B}_{i}(i=1,2,3,4)$; and denote $\Gamma_{h_{8}}^{k}$ the homoclinic cycle which surrounds $\tilde{D}_{k}$ and passes through saddle point $\tilde{E}_{j}$, where $j=3$, if $k=1,2 ; j=4$ if $k=3,4$.

Therefore, when $(\delta, \mu, r, k, n) \in \Sigma_{2}$ varies from $\Sigma_{3}$ to an appropriate direction, $\tilde{\Gamma}_{h_{3}}^{i}$ and $\tilde{\Gamma}_{h_{8}}^{i}, i=1,2,3,4$, are broken, and an unstable limit cycle occurs in the inner neighbourhood of each $\tilde{\Gamma}_{h_{3}}^{i}$ and $\tilde{\Gamma}_{h_{8}}^{i}(i=1,2,3,4)$; and each limit cycle occurred surrounds the other two small amplitude limit cycles inside each of $\tilde{\Gamma}_{h_{3}}^{i}$ and $\tilde{\Gamma}_{h_{8}}^{i}$ respectively.

In order to know the direction of the variable $(\delta, \mu, r, k, n)$ for which $\tilde{\Gamma}_{h_{3}}^{i}$ and $\tilde{\Gamma}_{h_{8}}^{i}$ generate limit cycles, let us consider $\Gamma_{h}: x^{2} / 2+y^{2} / 2-5 x^{4} / 8-5 y^{4} / 32+x^{6} / 6+$ $y^{6} / 96=h$. From which we have $\partial y / \partial h=1 /\left(y\left(1-y^{2} / 2\right)\left(1-y^{2} / 8\right)\right)$, then

$$
\begin{aligned}
M^{\prime}(h) & =\oint_{\Gamma_{h}}\left(\delta+\mu x^{2}+3 r y^{2}+k x^{4}+3 n x^{2} y^{2}+5 y^{4}\right)(\partial y / \partial h) \mathrm{d} x \\
& =\oint_{\Gamma_{h}} \frac{\left(\delta+\mu x^{2}+3 r y^{2}+k x^{4}+3 n x^{2} y^{2}+5 y^{4}\right)}{y\left(1-y^{2} / 2\right)\left(1-y^{2} / 8\right)} \mathrm{d} x \\
& =\oint_{\Gamma_{h}}\left(\delta+\mu x^{2}+3 r y^{2}+k x^{4}+3 n x^{2} y^{2}+5 y^{4}\right) \mathrm{d} t \\
& =\oint_{\Gamma_{h}}\left(\partial P_{\varepsilon} / \partial x+\partial Q_{\varepsilon} / \partial y\right) \mathrm{d} t .
\end{aligned}
$$

Since $\left.\left(\partial P_{\varepsilon} / \partial x+\partial Q_{\varepsilon} / \partial y\right)\right|_{B_{i}}>0$, as $(\delta, \mu, r, k, n)=(\bar{\delta}, \bar{\mu}, \bar{r}, \bar{k}, \bar{n})$, it may assume that when $(\delta, \mu, r, k, n) \in \Sigma_{2},\left.\left(\partial P_{\varepsilon} / \partial x+\partial Q_{\varepsilon} / \partial y\right)\right|_{B_{i}}>0$. Assume that $\Gamma_{h_{3}}$ is a heteroclinic loop surrounding $A_{i}$, and tending to $B_{i}(i=1,2,3,4)$ as $t \rightarrow \pm \infty$, thus, $M^{\prime}\left(h_{3}\right)=\oint_{\Gamma_{h_{3}}}\left(\partial P_{\varepsilon} / \partial x+\partial Q_{\varepsilon} / \partial y\right) \mathrm{d} t>0$; but $M\left(h_{3}\right)=0$, so there exists a left neighbourhood $\left(h_{3}-\Delta h, h_{3}\right)$ of $h_{3}$, such that $M(h)<0, M^{\prime}(h)>0$ as 
$h \in\left(h_{3}-\Delta h, h_{3}\right)$.

Now let $D=\Sigma_{2} \cap\left\{(\delta, \mu, r, k, n) \mid \oint_{\Gamma_{h_{3}}}\left(\delta y+\mu x^{2} y+r y^{3}+k x^{4} y+n x^{2} y^{3}+y^{5}\right) \mathrm{d} x>0\right\}$, for $(\delta, \mu, r, k, n) \in D$, then there exists a $h^{*} \in\left(h_{3}-\Delta h, h_{3}\right)$ such that $M\left(h^{*}\right)<0$, $M\left(h_{3}\right)>0$, so there exists a $h_{16} \in\left(h^{*}, h_{3}\right)$ such that $M\left(h_{16}\right)=0$, and $M^{\prime}\left(h_{16}\right)>0$, where $M\left(h_{16}\right)$ is an integral over a connected component $\Gamma_{h_{16}}^{\prime}$ of $\Gamma_{h_{16}}$ which is a closed orbit of (1.2) surrounding singular point $A_{i}(i=1,2,3,4)$. Therefore there is an unstable limit cycle of (1.4) in the neighbourhood of $\Gamma_{h_{16}}^{\prime}$.

Similarly, when $(\delta, \mu, r, k, n)=(\bar{\delta}, \bar{\mu}, \bar{r}, \bar{k}, \bar{n})$, we have $M\left(h_{8}\right)=0, M^{\prime}\left(h_{8}\right)=$ $\oint_{\Gamma_{h_{8}}}\left(\partial P_{\varepsilon} / \partial x+\partial Q_{\varepsilon} / \partial y\right) \mathrm{d} t>0$, so there exists a right neighbourhood $\left(h_{8}, h_{8}+\right.$ $\Delta h$ )of $h_{8}$, such that $M(h)>0, M^{\prime}(h)<0$ as $h \in\left(h_{8}, h_{8}+\Delta h\right)$. Now let $V=$ $D \cap\left\{(\delta, \mu, r, k, n) \mid \oint_{\Gamma_{h}}\left(\delta y+\mu x^{2} y+r y^{3}+k x^{4} y+n x^{2} y^{3}+y^{5}\right) \mathrm{d} x>0\right\}$, then for $(\delta, \mu, r, k, n) \in V$, there exists a $h_{17} \in\left(h_{8}, h_{8}+\Delta h\right)$ such that $M\left(h_{17}\right)=0$, $M^{\prime}\left(h_{17}\right)>0$, where $M\left(h_{17}\right)$ is an integral over a connected component $\Gamma_{h_{17}}^{\prime}$ of $\Gamma_{h_{17}}$ which is a closed orbit of (1.2) surrounding singular point $D_{i}(i=1,2,3,4)$. Therefore there exists an unstable limit cycle of (1.4) in the neighbourhood of $\Gamma_{h_{17}}^{\prime}$.

Summing up the above discussion, when $(\delta, \mu, r, k, n) \in V$, we conclude that system (1.4) has 29 limit cycles with their distributions as shown in Figure 3 . Theorem 1.1 is proved.

\section{References}

[1] L. Chen and M. Wang, 'The number and relation position of limit cycles for quadratic system', Acta Math. Sinica 22 (1979), 751-758 (Chinese).

[2] S. N. Chow, C. Li and D. Wang, Normal forms and bifurcation of planar vector fields (Cambridge University Press, 1994).

[3] H. Dulac, 'Sur les cycles limits', Bull. Soc. Math. France 51 (1923), 45-188.

[4] D. Hilbert, 'Mathematische Problems', Arch. Math. Phys. 3 (1901), 44-63, 213-237.

[5] J. $\mathrm{Li}$ and $\mathrm{Q}$. Huang, 'Bifurcations of limit cycles forming compound eyes in the cubic system', Chinese Ann. Math. 813 (1987), 391-403.

[6] S. Shi, 'An example of a quadratic system having at least four limit cycles', Scientia Sinica 11 (1979), 1051-1056 (Chinese).

[7] Y. Ye, Theory of limit cycles (Shanghai Scientific and Technology Press, 1984).

[8] Z. Zhang, Qualitative theory of differential equations (Science Press, Beijing, China, 1985).

[9] H. Zoladek, 'Eleven small limit cycles in a cubic field', Nonlinearity 8 (1995), 843-860.

Department of Mathematics

Fuzhou University

Fuzhou 350002

China

e-mail: gnchen@fzu.edu.cn 
